\title{
Seguidor Solar de Dos Ejes para un Horno Solar
}

\author{
Gabriel Villeda $^{(1)}$, Alejandro Castañeda ${ }^{(2)}$, José T. Vega ${ }^{(1)}$ y Jorge Pineda ${ }^{(1)}$ \\ (1) Centro de Investigación en Ciencia Aplicada y Tecnología Avanzada Querétaro, Instituto \\ Politécnico Nacional, Cerro Blanco 141, Colinas del Cimatario, 76090 Qro, Querétaro-México \\ (e-mail: gvilledam9300@ipn.mx, trinidad_vega66@yahoo.com.mx, arqjpp@yahoo.com) \\ (2) Ingeniería en Telemática, Dirección de Tecnologías de Información y Comunicación, \\ Universidad Politécnica de Querétaro, Carretera Estatal 420, El Rosario, El Marqués, Querétaro, \\ 76240, México (e-mail: dr.alejandrocm@gmail.com)
}

Recibido May. 11, 2010; Aceptado Jul. 13, 2010; Versión Final recibida Ago. 16, 2010

\section{Resumen}

Se presenta el diseño y fabricación de un seguidor solar de dos ejes (rotación-elevación), el cual controla un helióstato de un horno solar para la cocción de tabiques de arcilla. El sistema trabaja con motores controlados desde una computadora personal. El algoritmo para el seguidor solar se desarrolló en un lenguaje de programación visual, calcula los ángulos de seguimiento primario y secundario del helióstato y los despliega en una pantalla. El microcontrolador está programado para controlar el funcionamiento de los motores a pasos, los cuales mueven el helióstato del horno solar. El seguimiento primario y secundario es el mismo para los equinoccios, mientras que para los solsticios son diferentes debido a que durante el verano en el hemisferio norte existe mayor altura solar que en invierno. El seguidor solar permite una captación más eficiente de la radiación solar debido a que sigue minuto a minuto el movimiento aparente del Sol.

Palabras clave: seguidor solar, horno solar, helióstato, seguimiento primario, seguimiento secundario

\section{Two-Axis Sun Tracking System for a Solar Furnace}

\begin{abstract}
The design and manufacture of a two-axis sun tracking system (rotation-elevation), which controls a heliostat of a solar furnace for clay brick firing is presented. The system works with motors controlled through a personal computer. The algorithm for the sun tracking system was developed in a visual programming language, calculates the primary and secondary tracking angles of the heliostat and shows them in a screen. The microcontroller is programmed to control the step driver engines, which move the heliostat of the solar furnace. The primary and secondary tracking is the same for the equinoxes, whereas for the solstices are different because the solar altitude is greater in summer than in winter in the northern hemisphere. The sun tracking system permits a more efficient capture of the solar radiation since it continuously follows the apparent movement of the Sun.
\end{abstract}

Keywords: sun tracking system, solar furnace, heliostat, primary tracking, secondary tracking 


\section{INTRODUCCIÓN}

La elaboración artesanal de tabiques de arcilla (ladrillo rojo recocido) para la industria de la construcción en México sigue siendo una actividad económica importante, de la cual dependen muchas familias y que a pesar de los nuevos materiales de construcción se sigue utilizando en grandes cantidades.

La mayor parte de la producción se obtiene de manera artesanal; para la cocción de los tabiques se emplean hornos tradicionales los cuales son altamente contaminantes. Los principales combustibles utilizados son desechos industriales y en los lugares donde no hay industria, se queman desechos domésticos (basura), neumáticos o madera, contaminando el medio ambiente gravemente.

Por esta razón el CICATA-IPN Querétaro está desarrollando un horno solar de alta temperatura para la cocción de tabiques de arcilla, el cual alcanzará temperaturas de $1000^{\circ} \mathrm{C}$.

El prototipo del horno solar tiene una capacidad para 10 piezas, está constituido por tres componentes básicos: el helióstato, el concentrador parabólico fuera de eje y la cámara de cocción (Chen et alt., 2001; Chen et alt., 2002), tal como se muestra en la Fig. 1.

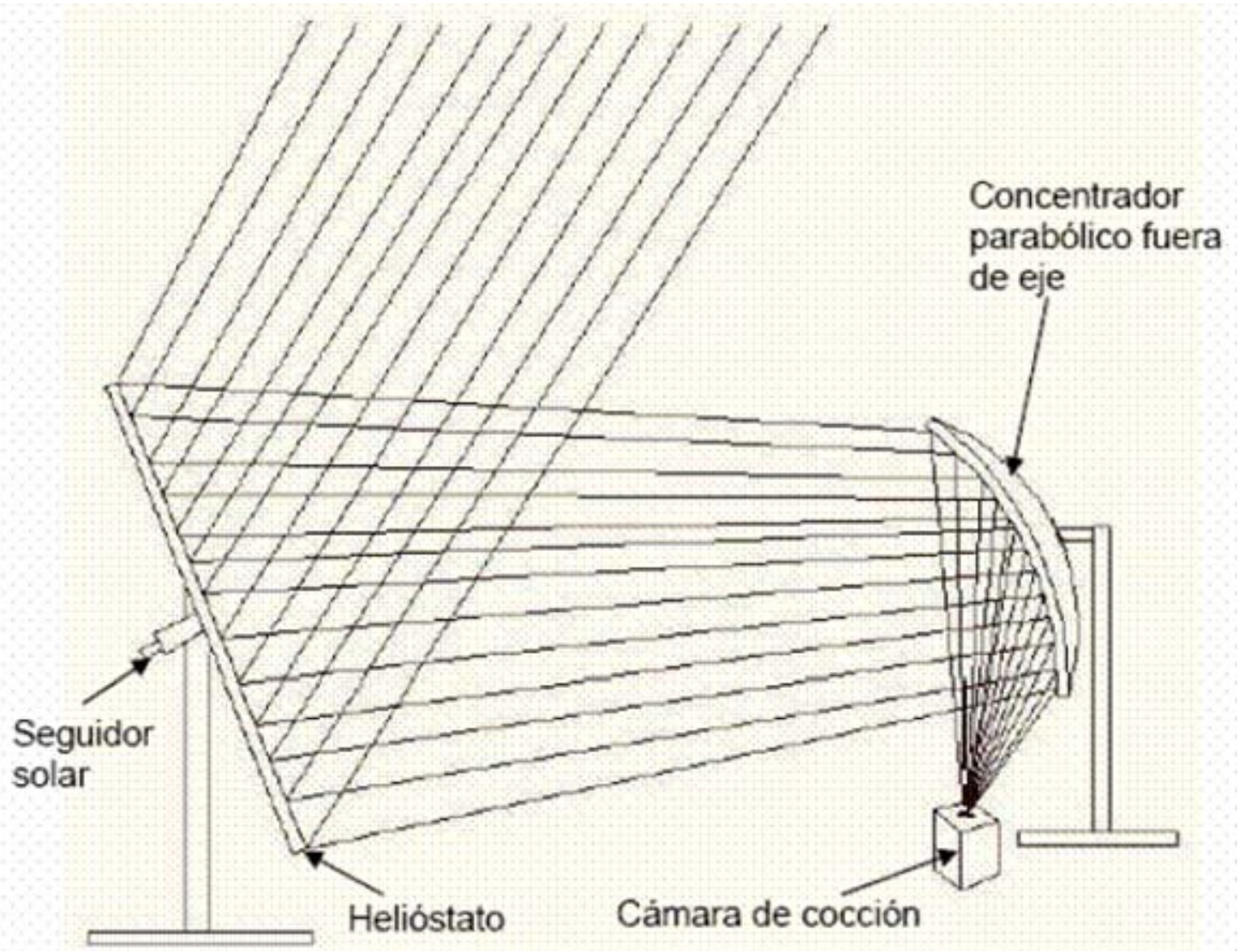

Fig. 1: Principales Componentes del Horno Solar.

El helióstato está formado por 9 espejos de $1 \mathrm{~m}^{2}$, tiene un seguidor solar de dos ejes cuyo propósito es lograr un aprovechamiento máximo de la radiación directa del Sol. El sistema completo del seguidor solar permite controlar los motores de seguimiento primario y secundario del helióstato, reflejando los rayos del Sol a un concentrador parabólico fuera de eje y este a su vez los dirige a la cámara de cocción.

Existen diversos tipos de seguidor solar, Huang y Sun (2007) presentaron el estudio de un seguidor solar de un eje y tres posiciones, el cual ajusta la posición de un módulo fotovoltaico únicamente en tres ángulos fijos: mañana, mediodía y tarde. 
El seguidor solar de dos ejes más comúnmente empleado es el azimut-elevación, Bakos (2006) diseñó y construyó un seguidor solar de dos ejes para mejorar la eficiencia de un concentrador parabólico comparándolo con una superficie fija inclinada a $40^{\circ}$; Duarte et al. (2010) desarrollaron un seguidor solar de dos ejes basado en mapas solares controlado por un microcontrolador de baja potencia; Sarker et al. (2010) presentaron el diseño, construcción y estudio experimental de un seguidor de dos ejes (azimut-polar) que consiste de un sensor y un microcontrolador que controlan dos motores a pasos; Jones (2010) desarrolló un sistema que permite seguir el movimiento aparente del Sol con una serie de paneles.

Los helióstatos con seguidor solar azimut-elevación generan aberraciones ópticas, la aberración incrementa el tamaño de la mancha solar reduciendo el flujo de calor en el receptor (Chen et alt., 2004). Para realizar una corrección de la aberración en el seguidor azimut-elevación, cada espejo esclavo debe tener movimiento con dos grados de libertad sobre el pivote que los soporta, lo cual incrementa el costo debido a que se requieren dos motores por espejo, es decir, se requieren $2 \mathrm{x}$ $(\mathrm{M} \times \mathrm{N})$, motores, lo cual significa que el helióstato de 9 espejos (acomodados en 3 filas y 3 columnas) que se propone en el diseño del horno solar, requiere de 18 motores.

Si se utiliza un seguidor rotación-elevación se requiere de $M+N$ motores, es decir, únicamente 6 motores, debido a que se necesita un motor para controlar cada fila o columna en lugar de dos motores para cada espejo, lo que representa un ahorro económico bastante importante. El espejo maestro está fijo al centro, los espejos esclavos tienen dos grados de libertad y están alrededor del espejo maestro. Cada espejo esclavo se mueve de manera angular sobre un pivote para reflejar los rayos del Sol al mismo punto fijo (Aliman et alt., 2007) Por esta razón se decide utilizar un seguidor rotación-elevación. La Fig. 2 muestra las diferencias que existen entre el seguidor azimut-elevación y el seguidor rotación-elevación.

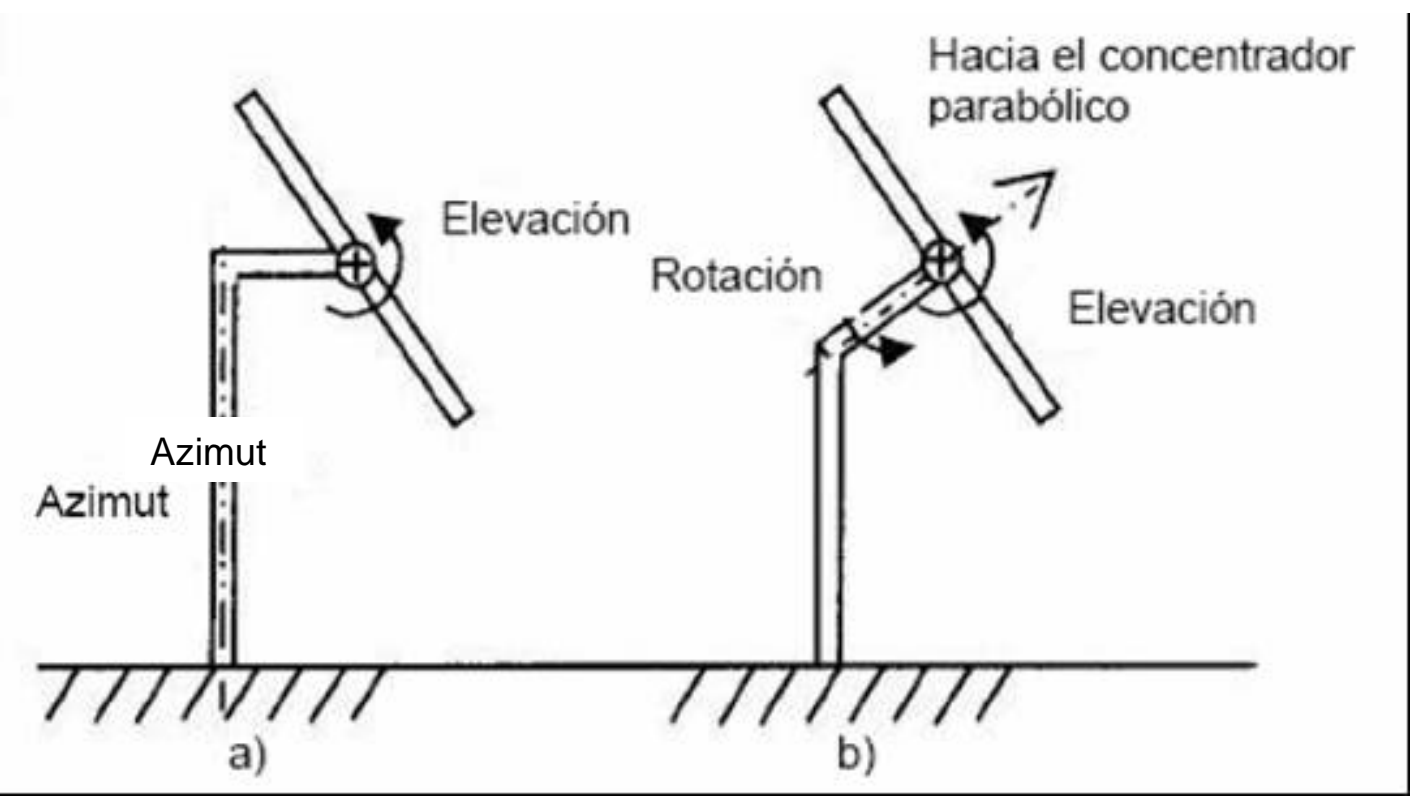

Fig. 2: Diagrama Esquemático que muestra la Diferencia entre los Dos Sistemas de Seguimiento. a) Azimut-Elevación, b) Rotación-Elevación. (Chen et alt., 2004).

El seguidor solar se desarrolló en un lenguaje de programación visual, el cual calcula los ángulos de seguimiento primario y secundario del helióstato y los despliega en una pantalla

\section{DESARROLLO DEL SISTEMA PARA SEGUIDOR SOLAR}

El movimiento del helióstato está dividido en dos partes: el movimiento global del marco con el espejo maestro el cual se denomina seguimiento primario y el movimiento local de los espejos esclavos llamado seguimiento secundario (Fig. 3). 
En el seguimiento primario, el ángulo de rotación se genera cuando el helióstato rota alrededor del eje $T_{1} T_{2}$ y el ángulo de elevación se produce cuando el helióstato rota alrededor del eje $U_{1} U_{2}$.

El seguimiento secundario se emplea para la corrección de aberraciones. Los espejos esclavos están agrupados en filas y columnas, los espejos en la misma fila o columna son movidos por el mismo motor. La Fig. 3 representa un helióstato de 3 filas y 3 columnas, el espejo maestro está al centro del helióstato en la fila 2 - columna 2 y los espejos esclavos son los ocho espejos alrededor.

El sistema del seguidor solar está desarrollado en un lenguaje de programación visual, se realizan una serie de cálculos para obtener los ángulos de seguimiento primario y secundario del helióstato, tal como se indica en el algoritmo de la Fig. 4.

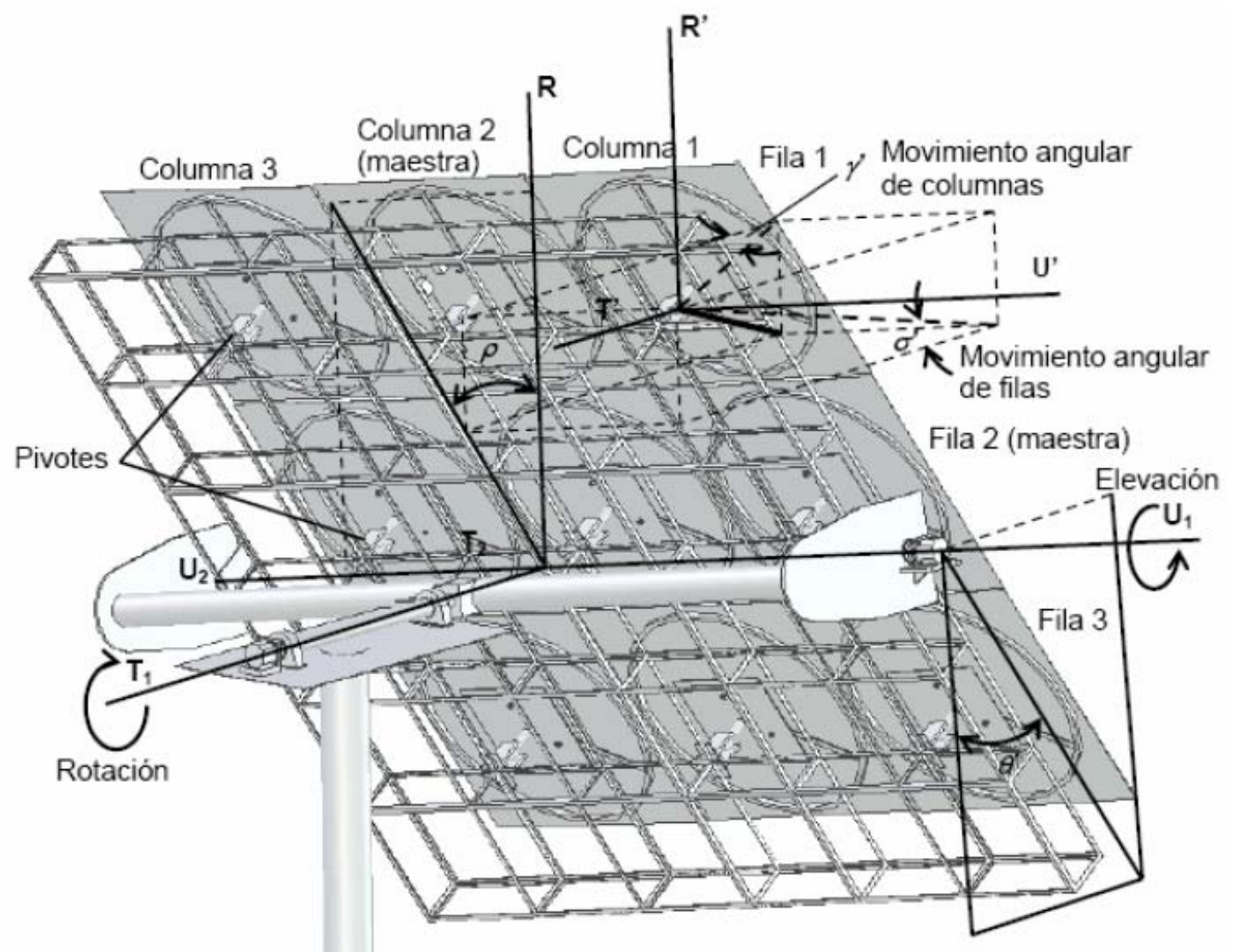

Fig. 3: Seguimiento Primario (Rotación-Elevación) y Seguimiento Secundario (Movimiento de Filas y Columnas).

\section{Parámetros de Entrada}

Se toma del sistema operativo de la computadora (ordenador) la fecha $(F)$ y la hora estándar $(H E)$; también se debe considerar la longitud $(L O)$ y latitud $(\phi)$ del lugar donde se localiza el seguidor solar, en este caso el sistema está desarrollado para la ciudad de Querétaro en México con longitud oeste de $100^{\circ} 23^{\prime} 34^{\prime \prime}$ y latitud norte de $20^{\circ} 35^{\prime} 15^{\prime \prime}$, sin embargo el sistema se puede adaptar para cualquier parte del mundo.

\section{Cálculos}

La hora estándar en decimales (HED), se calcula con la siguiente ecuación:

$H E D=$ Horas $+\frac{\left(\text { Minutos }+\frac{\text { Segundos }}{60}\right)}{60}$ 


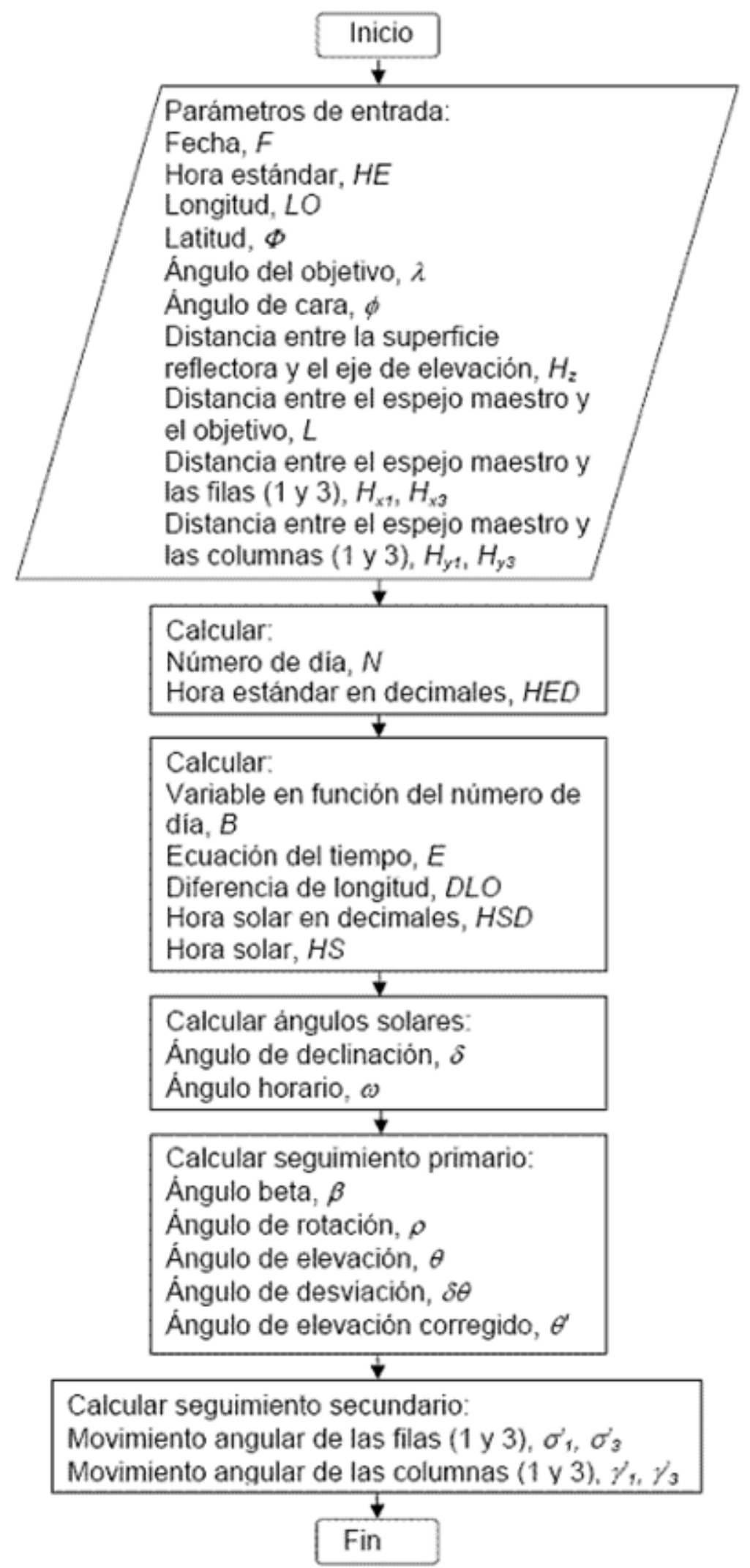

Fig. 4: Algoritmo del Seguidor Solar.

La hora solar (HSD) es la que se utiliza para calcular los ángulos solares, no coincide con la hora estándar local. Duffie y Beckman (1991), mencionan que es necesario convertir la hora estándar aplicando dos correcciones. Primero, existe una corrección constante por la diferencia de longitud $(D L O)$ entre el meridiano donde se ubica el observador $\left(L_{1 o c}\right)$ y el meridiano estándar en el cual está basado la hora local $\left(L_{s t}\right)$ : 


$$
D L O=\frac{4\left(L_{s t}-L_{l o c}\right)}{60}
$$

La segunda corrección es la ecuación del tiempo $(E)$, la cual considera las perturbaciones de la rotación de la Tierra:

$$
E=229.2(0.000075+0.001868 \cos B-0.032077 \operatorname{sen} B-0.014615 \cos 2 B-0.04089 \operatorname{sen} 2 B)
$$

donde:

$B=(N-1) \frac{360}{365}$

La hora solar en decimales (HSD) se calcula con la siguiente ecuación:

$H S D=H E D+D L O+E$

\section{Cálculo de Ángulos Solares}

El ángulo de declinación de la Tierra $(\delta)$ define la posición angular del Sol al mediodía solar, es decir, en el momento en que el Sol está más alto en el firmamento con respecto al plano del ecuador. Este parámetro depende del día del año, puede calcularse con la expresión:

$$
\delta=23.45 \operatorname{sen}\left(360 \cdot \frac{284+N}{365}\right)
$$

El ángulo horario $(\omega)$ es igual a cero al medio día solar y adquiere un valor de $15^{\circ}$ de longitud por cada hora, siendo negativo en las mañanas y positivo por las tardes, se calcula con la ecuación:

$\omega=15(H S D-12)$

\section{Cálculo del Seguimiento Primario}

El ángulo de rotación $(\rho)$ se genera cuando el helióstato rota alrededor del eje $T_{1} T_{2}$, el plano de reflexión está en posición horizontal en las mañanas y en posición vertical al medio día, se determina con la ecuación:

$$
\begin{aligned}
& \rho=\operatorname{sen}^{-1}\left\{\frac{\cos \delta \cos \omega \operatorname{sen} \phi \operatorname{sen} \Phi-\cos \delta \operatorname{sen} \omega \cos \phi-\operatorname{sen} \delta \operatorname{sen} \phi \cos \Phi}{\cos \beta}\right\} \\
& \beta=\operatorname{sen}^{-1}\left\{\begin{array}{l}
\cos \delta \cos \omega(-\operatorname{sen} \lambda \cos \Phi+\cos \lambda \cos \phi \operatorname{sen} \Phi) \\
+\cos \delta \operatorname{sen} \omega \cos \lambda \operatorname{sen} \phi \\
-\operatorname{sen} \delta(\operatorname{sen} \lambda \operatorname{sen} \Phi-\cos \lambda \cos \phi \cos \Phi)
\end{array}\right\}
\end{aligned}
$$

El ángulo de elevación $(\theta)$ se produce cuando el helióstato rota alrededor del eje $\cup_{1} U_{2}$, se determina con la ecuación (Chen et alt., 2001):

$\theta=\frac{1}{2}\left(\frac{\pi}{2}-\beta\right)$

Sin embargo, la superficie reflectora del espejo y el eje de elevación están separados debido al espesor de los espejos y la estructura que los soporta. Por lo tanto, es necesario realizar una corrección del ángulo de elevación con la siguiente ecuación (Chen et alt., 2002): 
$\theta^{\prime}=\theta-\delta \theta$

$\delta \theta=\frac{1}{2} \tan ^{-1}\left(\frac{H_{z} \operatorname{sen} \theta}{L-H_{z} \cos \theta}\right)$

\section{Cálculo del Seguimiento Secundario}

El seguimiento secundario se emplea para la corrección de aberraciones. Los espejos esclavos están agrupados en filas y columnas, los espejos en la misma fila o columna serán movidos por el mismo motor. La Fig. 3 representa un helióstato de 3 filas y 3 columnas, el espejo maestro está en la fila 2 y los espejos esclavos en 1 y 3.

Los espejos esclavos acomodados en las filas tienen que rotar sobre los pivotes un ángulo $\sigma^{\prime}$ para garantizar que los rayos del Sol lleguen al mismo punto, se calcula con la ecuación (Chen et alt., 2002):

$\sigma^{\prime}=\frac{1}{2} \tan ^{-1}\left(\frac{H_{x} \cos (2 \delta \theta) \cos (\theta+\delta \theta)}{H_{x} \cos (2 \delta \theta) \operatorname{sen}(\theta+\delta \theta)+L-H_{z} \cos (\theta-\delta \theta)}\right)$

Los espejos esclavos acomodados en las columnas tienen que rotar sobre un ángulo $\gamma^{\prime}$ para que los rayos del Sol lleguen al objetivo (Chen et alt., 2002):

$\gamma^{\prime}=\frac{1}{2} \tan ^{-1}\left(\frac{H_{y}}{L \cos (\theta-\delta \theta)-H_{z}}\right)$

El sistema trabaja con motores controlados desde una computadora personal, que es programada para calcular la hora solar en tiempo real, así como para obtener los ángulos de seguimiento primario y secundario del helióstato.

El algoritmo para el seguidor solar se desarrolló en un lenguaje de programación visual; donde la computadora personal, usando datos codificados por una interfaz de comunicación serial, se interconecta con el seguidor a través de un sistema microprocesador.

El microcontrolador está programado con una secuencia de datos para controlar el funcionamiento de los motores, los cuales son coordinados en función de las coordenadas recibidas. Con una etapa de potencia se obtienen las corrientes especificadas a los motores. El sistema completo del seguidor solar permite controlar los movimientos de seguimiento primario y secundario del helióstato, el sistema completo es autónomo, la Fig. 5a ilustra los componentes de la interfaz eléctrica (sistema de alimentación) y la Fig. 5b la interfaz electrónica (módulo de control) del seguidor solar.

\section{RESULTADOS}

El seguidor solar se desarrolló en un lenguaje de programación visual, el cual calcula los ángulos de seguimiento primario y secundario del helióstato descritos en el algoritmo de la figura 4 y los despliega en una pantalla, tal como se muestra en la figura 6. 

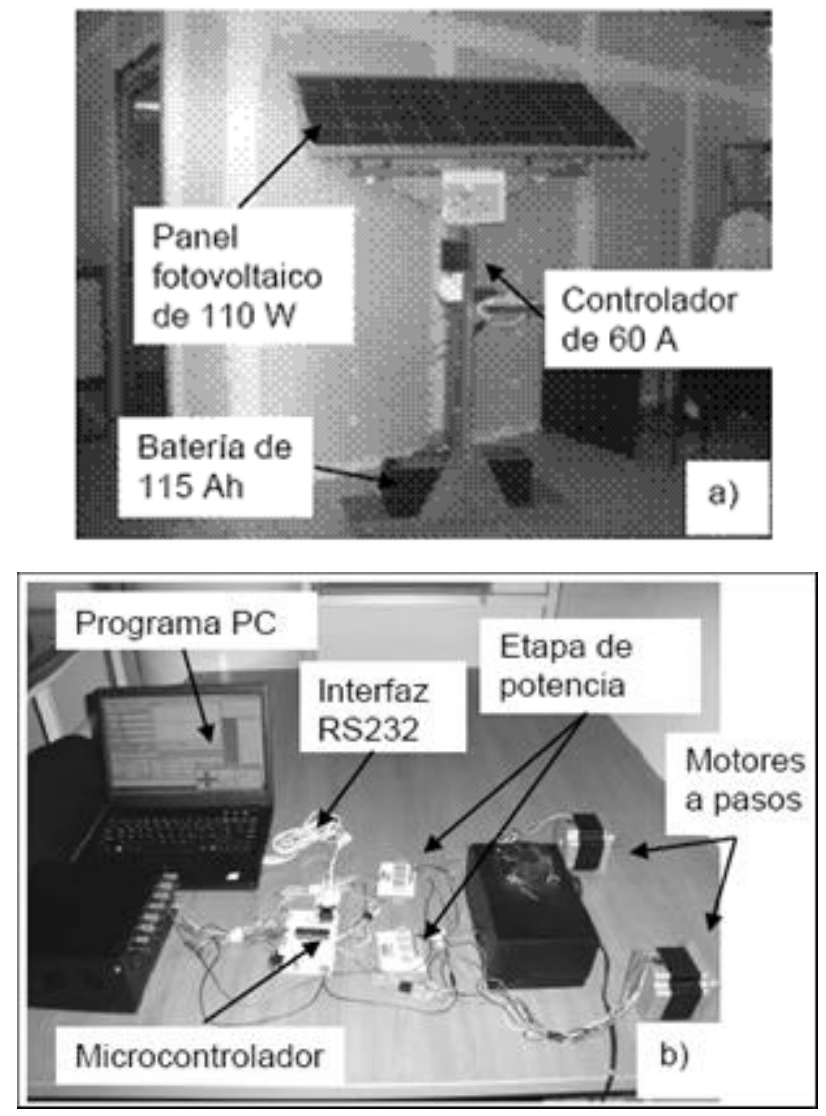

Fig. 5: Componentes del Seguidor Solar. a) Interfaz Eléctrica, b) Interfaz Electrónica.

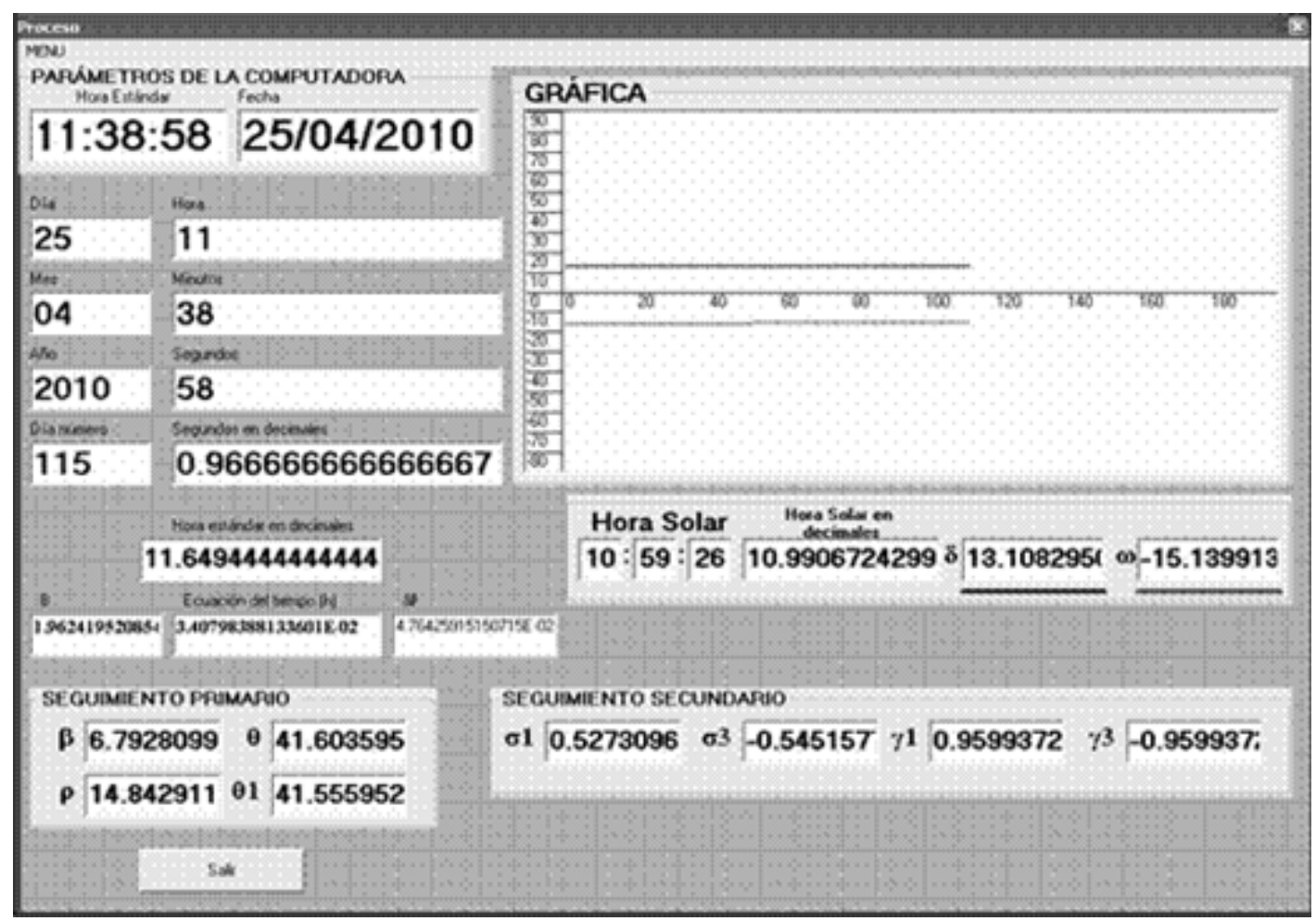

Fig. 6: Cálculo de los Ángulos con el Programa de Computación. 
Utilizando las ecuaciones (8) y (11) se pueden construir las gráficas del ángulo de rotación $\rho$ y elevación corregido $\theta$ para cada día del año. La Fig. 7 muestra las gráficas para los equinoccios de primavera y otoño cuando el ángulo de declinación es $0^{\circ}$, y para los solsticios de verano e invierno cuando el ángulo de declinación tiene su valor máximo y mínimo, $+23.45^{\circ}$ y $-23.45^{\circ}$ respectivamente.

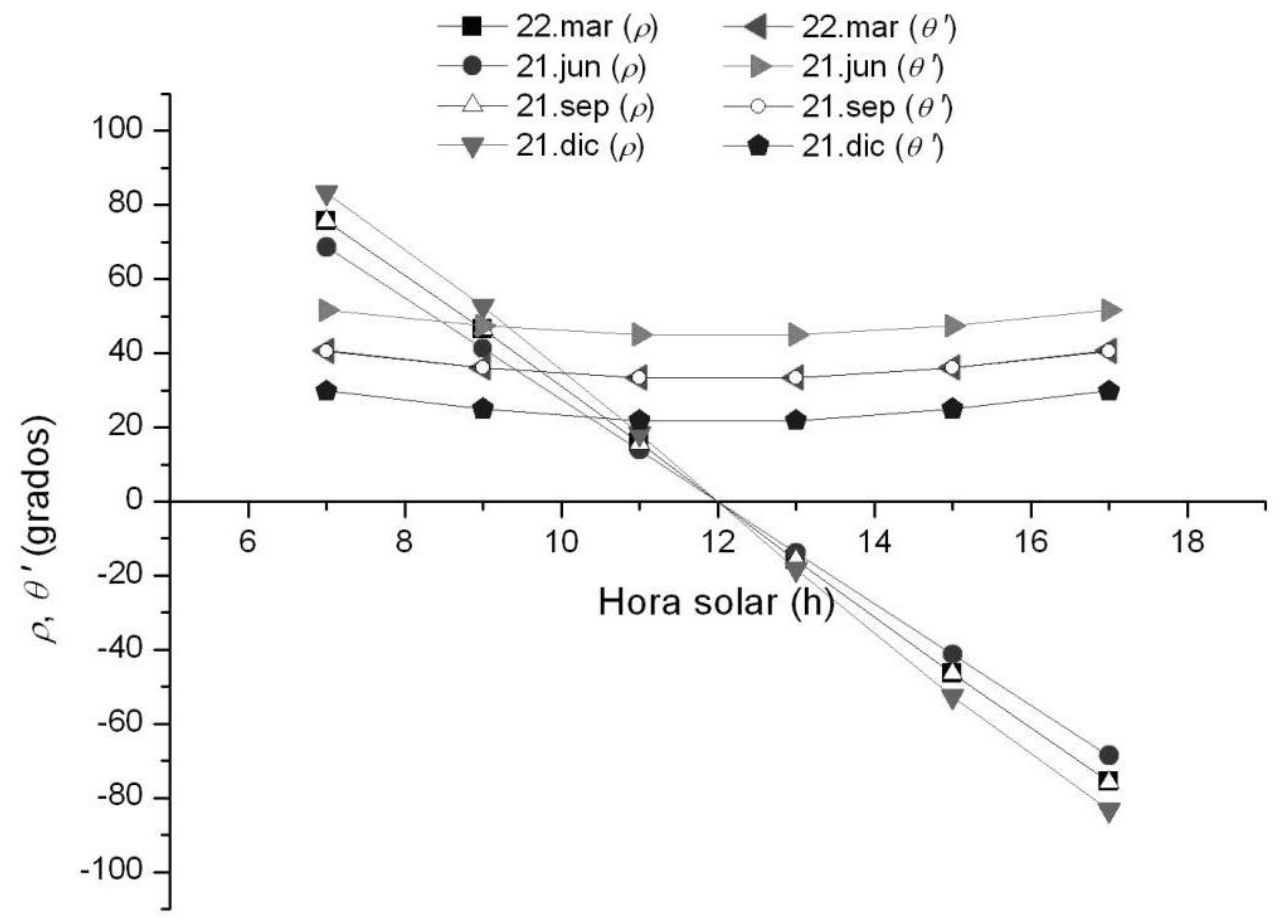

Fig. 7: Gráfica del Ángulo de Rotación $\rho$ y Elevación Corregido $\theta$ ’ del Helióstato.

La Fig. 8 muestra el movimiento angular de las filas $\sigma$ en función de la hora solar para los equinoccios y los solsticios, cuando las filas están localizadas a $H_{x}=+1.02 \mathrm{~m}, H_{x}=-1.02 \mathrm{~m}$ y $L=$ $30 \mathrm{~m}$. La Fig. 8 también muestra el movimiento angular de las columnas $\gamma$ cuando las filas están localizadas a $H_{y}=+1.02 \mathrm{~m}, H_{y}=-1.02 \mathrm{~m}$ y $L=30 \mathrm{~m}$.

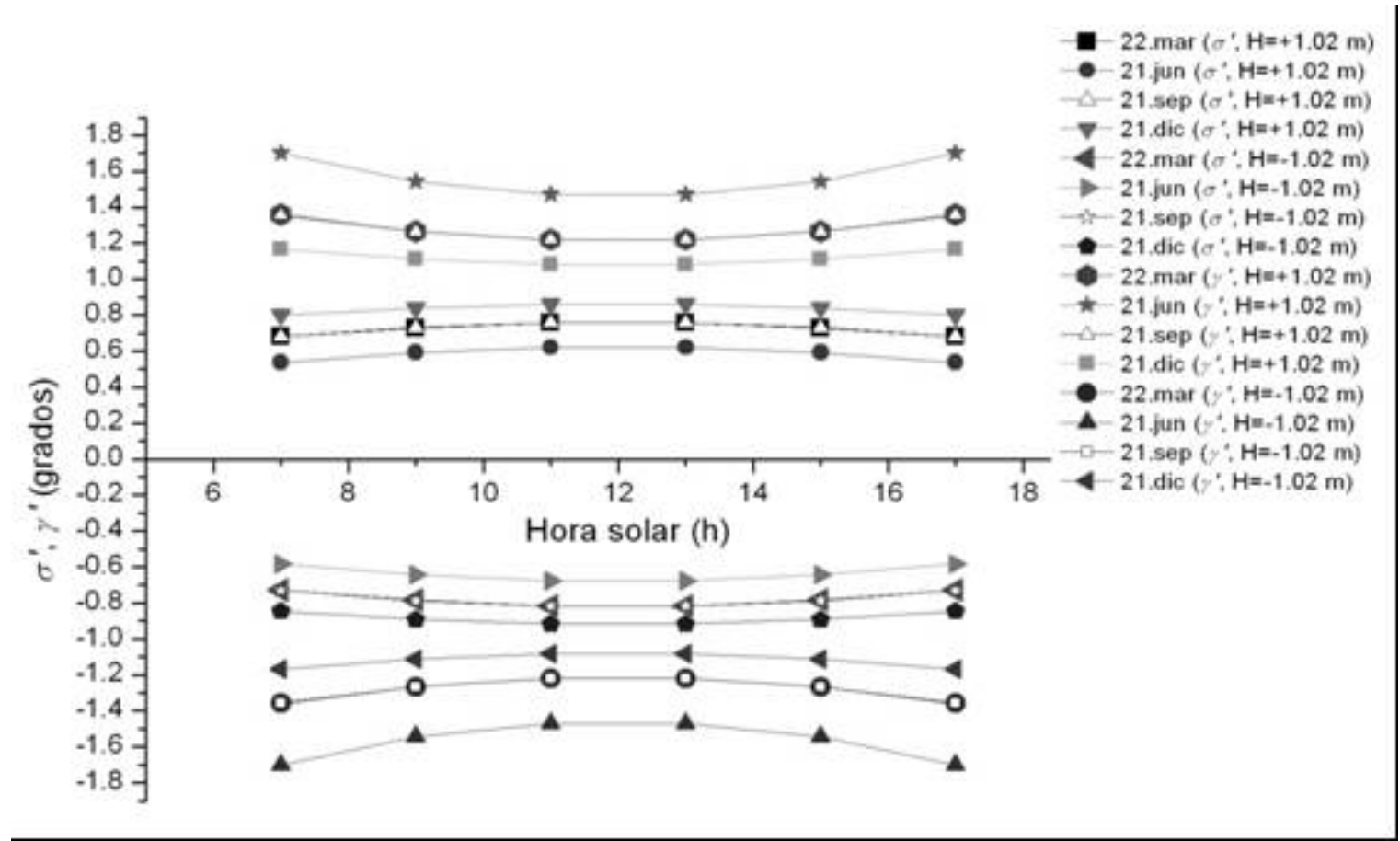

Fig. 8: Gráfica del Movimiento Angular de las Filas $\sigma^{\prime}$ y las Columnas $\gamma^{\prime}$. 
La figura 9a muestra un pequeño prototipo del helióstato con seguidor solar y la figura 9b el helióstato del horno solar para la cocción de 10 tabiques.
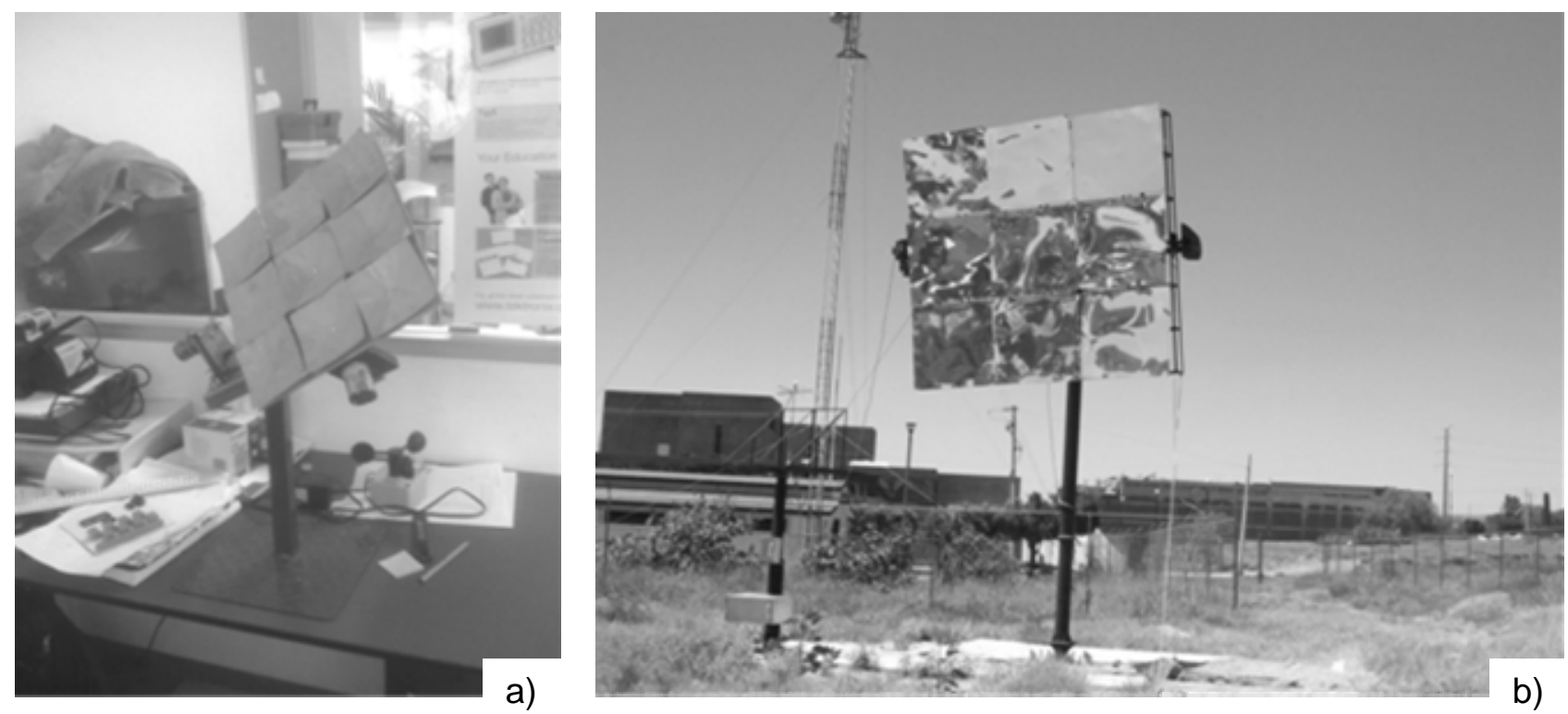

Fig. 9: a) Prototipo del Heliósıaıu, b) Helióstato del Horno Solar para la Cocción de 10 Tabiques.

\section{DISCUSIÓN}

La Fig. 7 muestra las gráficas del seguimiento primario. El movimiento de rotación del helióstato $\rho$ tiene un rango de $-90^{\circ}$ a $+90^{\circ}$ durante el día, las gráficas del ángulo de rotación para los equinoccios están encimadas debido a que el ángulo de declinación terrestre es $0^{\circ}$ para ambos casos. Las gráficas de rotación para los solsticios de verano e invierno muestran una diferencia máxima de aproximadamente $15^{\circ}$ para las primeras horas de la mañana y las últimas de la tarde. El movimiento de elevación corregido $\theta^{\prime}$ cubre diariamente un rango de $21.41^{\circ}$ a $32.42^{\circ}$ en invierno y de $44.74^{\circ}$ a $54.20^{\circ}$ en verano, las gráficas del ángulo de elevación corregido para los equinoccios están encimadas, mientras que para los solsticios existe una diferencia de $23.33^{\circ}$ a las $12: 00$ horas y de $21.78^{\circ}$ a las 6:00 y 18:00 horas (hora solar de la ciudad de Querétaro con latitud norte $\left.20^{\circ} 35^{\prime} 15^{\prime \prime}\right)$.

El movimiento secundario tiene desplazamientos menores a $2^{\circ}$ para filas y columnas, la Fig. 8 muestra las gráficas del seguimiento secundario. Las gráfica del movimiento angular de las filas $\sigma^{\prime}$ para los equinoccios están encimadas cuando $H_{x}=+1.02 \mathrm{~m}$ y $H_{x}=-1.02 \mathrm{~m}$, mientras que para los solsticios existe una diferencia de $0.23^{\circ}$ a las $12: 00$ horas y de $0.27^{\circ}$ a las 6:00 y 18:00 horas cuando $H_{x}=+1.02 \mathrm{~m}$ y $H_{x}=-1.02 \mathrm{~m}$.

La Fig. 8 también muestra las gráfica del movimiento angular de las columnas $\gamma$, para los equinoccios las gráficas están encimadas cuando $H_{y}=+1.02 \mathrm{~m}$ y $H_{y}=-1.02 \mathrm{~m}$, mientras que para los solsticios existe una diferencia de $0.38^{\circ}$ a las $12: 00$ horas y de $0.62^{\circ}$ a las 6:00 y 18:00 horas cuando $H_{y}=+1.02 \mathrm{~m}$ y $H_{y}=-1.02 \mathrm{~m}$.

\section{CONCLUSIONES}

De los argumentos presentados, de su discusión y de los antecedentes de la literatura expuestos a través del artículo, se pueden obtener las siguientes conclusiones principales:

i) Se desarrolló un sistema para el seguidor solar que sirve para controlar los motores de seguimiento primario y secundario de un helióstato. 
ii) El seguidor solar no requiere de sensores que detecten la posición del Sol; los motores son controlados desde una PC, la cual está programada para calcular en tiempo real la posición del helióstato, con el propósito de lograr un aprovechamiento máximo de la radiación directa del Sol.

iii) El movimiento de seguimiento primario del helióstato es el mismo para los equinoccios de primavera y otoño debido a que la altura solar es la misma, el movimiento de seguimiento secundario también es el mismo para ambos equinoccios. Existe una diferencia tanto para el movimiento de seguimiento primario como el secundario en ambos solsticios debido a que en el hemisferio norte en verano existe una mayor altura solar que en invierno.

iv) Una aplicación potencial que le da un valor agregado al sistema, es el uso de hornos solares para la cocción de ladrillos de arcilla. El horno solar tiene como objetivo eliminar la emisión de contaminantes hacia el aire, suelo y agua; ya que no se emplean desechos domésticos 0 industriales.

\section{NOMENCLATURA}

B Variable en función del número de día.

DLO Diferencia de longitud.

E Ecuación del tiempo.

HED Hora estándar en decimales.

$H S D$ hora solar en decimales.

$H_{x} \quad$ Distancia entre el centro de la fila maestra y el centro de la fila donde se encuentra el espejo esclavo a calcular (es positiva si la fila se encuentra arriba de la fila maestra y es negativa si la fila se encuentra abajo).

$H_{y} \quad$ Distancia entre el centro de la columna maestra y el centro de la columna donde se encuentra el espejo esclavo a calcular (es positiva si la columna se encuentra a la izquierda de la columna maestra y es negativa si la columna se encuentra a la derecha).

$\mathrm{H}_{z} \quad$ Distancia entre la superficie reflectora y el eje de elevación.

$L \quad$ Distancia entre el espejo maestro y el objetivo.

$L_{\text {loc }} \quad$ Meridiano donde se ubica el observador.

$L_{s t} \quad$ Meridiano estándar en el cual está basado la hora local.

$N \quad$ Número del día del año.

$\beta \quad$ Ángulo beta.

$\gamma \quad$ Movimiento angular de las columnas.

$\delta \quad$ Ángulo de declinación de la Tierra.

$\delta \theta \quad$ Ángulo de desviación.

$\theta \quad$ Ángulo de elevación.

$\theta^{\prime} \quad$ Ángulo de elevación corregido.

$\lambda \quad$ Ángulo del objetivo. Es igual a cero cuando el helióstato está al mismo nivel que el objetivo y tiene un valor por ejemplo de $15^{\circ}$ cuando el helióstato está abajo del objetivo.

$\rho \quad$ Ángulo de rotación.

$\sigma^{\prime} \quad$ Movimiento angular de las filas

$\Phi \quad$ Latitud.

$\phi \quad$ Ángulo de cara. Es igual a cero cuando el helióstato está al norte del objetivo y de $90^{\circ}$ cuando el helióstato se localiza al este del objetivo.

$\omega \quad$ Ángulo horario.

\section{AGRADECIMIENTOS}

Agradecemos a SIP-IPN y a la Universidad Politécnica de Querétaro por el apoyo económico y las facilidades otorgadas para el desarrollo de este proyecto. 


\section{REFERENCIAS}

Aliman, O., I. Daut, M. Isa y M.R. Adzman, Simplification of Sun Tracking Mode to Gain Concentration Solar Energy, American J. Applied Sciences: 4(3), 171-175 (2007).

Bakos, G.C., Design and Construction of a Two-Axis Sun Tracking System for Parabolic Trough Collector (PTC) Efficiency Improvement, Renewable Energy: 31(15), 2411-2421 (2006).

Chen, Y.T. y otros 14 autores, Non-Imaging Focusing Heliostat, Solar Energy: 71(3), 155-164 (2001).

Chen, Y.T. y otros ocho autores, Report of the First Prototype of Non-Imaging Focusing Heliostat and its Application in High Temperature Solar Furnace, Solar Energy: 72(6), 531-544 (2002).

Chen, Y.T. y otros ocho autores, Comparison of Two Sun Tracking Methods in the Application of a Heliostat Field, J. Solar Energy Engineering: 126, 638-644 (2004).

Duarte, F., P. Dinis-Gaspar y L. Carrilho-Gonçalves, Two Axis Solar Tracker Based on Solar Maps, Controlled by a Low-Power Microcontroller, International Conference on Renawable Energies and Power Quality, Granada, España, 23 al 25 de Marzo (2010).

Duffie, J.A. y W.A. Beckman, Solar Engineering of Thermal Process, $2^{a}$ edición, 11-12. John Wiley \& Sons, Wisconsin, USA (1991).

Huang, B.J. y F.S. Sun, Feasibility Study of One Axis Three Position Tracking Solar PV with Low Concentration Ratio Reflector, Energy Conversion \& Management: 48, 1273-1280 (2007).

C. Jones, Solar Tracking System, WO 2009/039556 A1, F24J 2/54 (2006.01), 2 de abril (2010).

Sarker, M.R.I., Md. Riaz-Pervez y R.A. Beg, Design, Fabrication and Experimental Study of a Novel Two-Axis Sun Tracker, International J. Mechanical \& Mechatronics Engineering: 10(1), 1318 (2010). 